\title{
Calidad De Vida En Las Familias Con Integrantes Con Discapacidad En Jalisco, Mexico: Una Aproximación Objetiva
}

\author{
Quality Of Life In Families With Members With Disabilities In Jalisco, \\ Mexico: A Strict Approach
}

\author{
Dr. Roberto Govela E.
}

Universidad Antropológica de Guadalajara

govelamilenium@gmail.com

\section{Resumen}

De acuerdo a Ardilla (2003:163) la calidad de vida de las personas con discapacidad posee aspectos objetivos y subjetivos que nos permiten valorarla. En ese sentido, el objetivo de este ensayo es medir los aspectos positivos. Para lograrlo, este trabajo se estructurará de la siguiente forma.

En un primer lugar se establecerá en la introducción, de qué forma se dio el cambio de abordar las repercusiones negativas de la presencia de un integrante con discapacidad en la familia, a la propuesta propositiva de abordar la calidad de vida de estas familias: Tema por cierto muy relevante, pero prácticamente no abordado en México. Una vez establecido esto, se establecerá, por un lado, el objetivo general de la indagación y por el otro, se describirá la propuesta metodológica del estudio, que tiene un enfoque cuantitativo.

En un segundo lugar se referirá el debate teórico existente acerca de la calidad de vida de las personas con discapacidad y que propuestas existen para analizarlo empíricamente.

En un tercer lugar se desarrollará la pesquisa, refiriendo por un lado, cual es el contexto estadístico de la discapacidad en el estado mexicano de Jalisco y por el otro, los datos duros, en cuanto a cuál es el acceso que tiene este colectivo al empleo, la salud, educación, vivienda y sus servicios: Indicadores que nos permitirán tener un acercamiento objetivo a la calidad de vida de las familias con integrantes con discapacidad.

Finalmente se harán las consideraciones finales.

Palabras claves: Calidad de vida, familias con integrantes con discapacidad, aspectos objetivos 


\begin{abstract}
According to Ardilla (2003: 163) life quality of people with disabilities have objective and subjective aspects that allow us to evaluate it. In that sense, the objective of this test is to measure the positive aspects. To achieve it, this work is structured as follow:

In the first place, the introduction is going to established as: How the way to react tow ard the negative consequences of a handicap member in the family has changed and propose a positive way to improve life quality in these families. A theme that is very relevant, but practically not addressed in Mexico. Once it will be approached more, it will establish, on one hand, the general objective of the inquiry and on the other hand, it will describe the methodological propose from the study, which has a quantitative focus.

In second place it will refer to the existent theoretical debate about the quality of life for people who have disabilities and there are proposes to analyse it empirically.

In a third place the inquest is going to be developed, referring on one hand, what the statistical context of disabilities in the state of Jalisco is and on the other, the hard data that has the access to employment, health, education, housing and services.

These indicators are going to allow us to have a closer approach to the objective of a quality life of the families who have a handicap member.

Finally the final considerations are going to be made.
\end{abstract}

Keywords: Quality of life, Families with members with disabilities y objective aspects

\section{Introducción}

De acuerdo a Córdova Andrade, Gómez Benito y Verdugo Alonso (2008, p. 370) la literatura científica hasta la década de los 90 muestra que los estudios en el área giraban en torno al impacto de la discapacidad en la familia (Weiss, Marvin \& Pianta, 1997; Browne \& Bramster, 1998; Rubio, Berg-Weger \& Tebb, 1999) (...)

De acuerdo a Schalock \&Verdugo (2002) se generó un cambio en donde se habló sobre la calidad de vida, no sólo de la persona con discapacidad, sino de la calidad de las familias que tiene un integrante con discapacidad (Córdova
Andrade, Gómez Benito y Verdugo Alonso, 2008 p. 370) En esta perspectiva se visualiza a la familia como una unidad de apoyo (Schalock \& Verdugo, 2002), trabajando en equipo con los profesionales de los servicios de atención para la persona con discapacidad y con otras familias, en la búsqueda de logros que respondan a sus necesidades reales y a sus expectativas (Córdova Andrade, Gómez Benito y Verdugo Alonso, 2008, p. 370)

Corraliza (1991) y posteriormente Ferreyra (2003) mencionan que la calidad de vida es un concepto que surge relacionado con el desarrollo de la sociedad de bienestar y que no sólo es visto como el grado de satisfacción subjetiva de un individuo, sino también como el nivel de recursos 
de los que dispone un individuo para poder dirigir su vida. (Muñoz Valdés, 2012)

Schalock (1999) menciona que el concepto de calidad de vida ha surgido bajo tres clasificaciones: (1) como un concepto de sensibilización, que aporta un punto de referencia y guía desde el sujeto, (2) como constructo social, usado como principio general para mejorar la calidad de vida de una persona y (3) como tema unificador, girando a su alrededor tanto el programa de rehabilitación como la evaluación del mismo. (Muñoz Valdés, 2012)

En el ámbito de la salud mental, Verdugo y Martin (2002) mencionan que la calidad de vida como concepto se encuentra asociado a enfoques que tienen como objetivo mejorar las condiciones de vida de las personas con trastornos psicológicos, considerando de esta manera sus necesidades individuales. (Muñoz Valdés, 2012)

A pesar de que el tema da la calidad de vida de las personas con discapacidad, es un abordaje recurrente en España, con autores de la talla de Verdugo Alonso $(2006,2008)$ y que es un tema que aparece en revista especializadas como lo ejemplifica el No. 63 de la Revista Iberoamericana de Educación, que dedicó una edición al tema (septiembre- diciembre, 2013) en México prácticamente no existe, ya que sigue prevaleciendo el abordaje de la discapacidad desde un abordaje estadístico (INEGI, 2010) o como una problemática meramente social (Antúnez
Farrugia y Balcazar de la Cruz, 2005)(Encuesta Nacional sobe Discriminación en México, 2010) Por ende, el abordaje de los factores que favorecen y/o obtaculizan el que estas familias vulnerables tengan una mejor calidad de vida, es una temática ampliamente justificable.

Por lo tanto, el objetivo de este ensayo, es establecer una aproximación a la calidad de vida de las familias que tienen un integrante con discapacidad en el estado mexicano de Jalisco, en base a la propuesta de Ardilla (2003:163)

Por ende, en enfoque de esta pesquisa es cuantitativo, ya que se recurrirán a datos cuantitativos que nos permitan establecer cuál es el acceso que tienen estas familias vulnerables al empleo, salud, educación, vivienda y servicios públicos, como una serie de parámetros que nos permitan determinar objetivamente su calidad de vida.

\section{La calidad de vida de las familias con integrantes con discapacidad}

\subsection{Conceptualización de calidad de vida y su medición}

Según Ardilla (2003, p.163) la calidad de vida es un estado de satisfacción general, derivado de la realización de las potencialidades de la persona. Posee aspectos subjetivos y aspectos objetivos. 
En esta definición hay varios aspectos que señalar, en primer lugar, que calidad de vida es un estado de satisfacción general, que surge de realizar las potencialidades que el individuo posee.

Realización personal y calidad de vida son dos aspectos que se han ligado, y que sin duda están altamente correlacionados.

En el concepto de calidad de vida se incluyen como aspectos subjetivos los siguientes:

1. Intimidad

2. Expresión emocional

3. Seguridad percibida

4. Productividad personal

5. Salud percibida

Para tener calidad de vida necesitamos sentirnos sanos, productivos, seguros, y ser capaces de expresar nuestras emociones y compartir nuestra intimidad.

Como aspectos objetivos la calidad de vida incluye:

1. Bienestar material

2. Relaciones armónicas con el ambiente

3. Relaciones armónicas con la comunidad

4. Salud objetivamente considerada

Como puede notarse, separamos salud percibida

y salud objetiva. Podemos sentimos saludables y no estarlo, o estarlo (salud objetiva) y no sentimos saludables.

La evaluación del concepto presenta una situación parecida. Para Dennis, Williams, Giangreco y Cloninger (1993) los enfoques de investigación de este concepto son variados, pero podrían englobarse en dos tipos: Enfoques cuantitativos y cualitativos, cuyo propósito es operacionalizar la calidad de vida. (Gómez-Vela, María; Sabeh, Eliana N., 2000)

En cuanto a los indicadores cuantitativos encontramos: 1) Sociales (se refieren a condiciones externas relacionadas con el entorno como la salud, el bienestar social, la amistad, el estándar de vida, la educación, la seguridad pública, el ocio, el vecindario, la vivienda, etc.); 2) Psicológicos (miden las reacciones subjetivas del individuo a la presencia o ausencia de determinadas experiencias vitales); y Ecológicos (miden el ajuste entre los recursos del sujeto y las demandas del ambiente) (Gómez-Vela, María; Sabeh, Eliana N., 2000)

En lo que respecta a los cualitativos, en estos se adopta una postura de escucha a la persona mientras relata sus experiencias, desafíos y problemas y cómo los servicios sociales pueden apoyarles eficazmente. (Gómez-Vela, María; Sabeh, Eliana N., 2000)

\subsection{Calidad de vida y discapacidad}

Considerando a Arostegui (1999) es importante destacar que los elementos clave en la calidad de vida de las personas con discapacidad están asociados a la capacidad de toma de decisiones, autocontrol, independencia y autonomía (Yohanina Andrea Muñoz Valdés, Yohanna del Pilar Poblete Toloza y Andrés Eduardo Jiménez Figueroa, 2012 p.211) 
En cuanto a cómo se concibe el concepto de calidad de vida de las familias con integrantes con discapacidad, Park, Turnbull y Turnbull (2002) precisaron que una familia experimenta calidad de vida cuando sus miembros tienen sus necesidades cubiertas, disfrutan de su vida juntos y cuentan con oportunidades para perseguir y alcanzar metas que son trascendentales para ellos. (Córdova Andrade, Gómez Benito y Verdugo Alonso, 2008, p. 370)

De acuerdo a Schalock y Verdugo (2002/2003), la calidad de vida individual es un estado deseado de bienestar personal que: (a) es multidimensional; (b) tiene propiedades etic y emic (Jenaro et al., 2005; Schalock et al, 2005); (c) tiene componentes objetivos y subjetivos; (d) está influenciada por factores personales y ambientales; y (e) incorpora la perspectiva de sistemas que engloba los múltiples ambientes que influyen en la vida de las personas (Keith y Bonham, 2005; Schalock, Gardner y Bradley, 2007; Schalock, Bonham y Verdugo, 2008; Schalock, Verdugo, Bonham, Fantova y van Loon, 2008, citados por L. E. Gómez; M. A. Verdugo; B. Arias y P. Navas, 2008 pp.192-193)

En la investigación de L. E. Gómez, M. A. Verdugo, B. Arias y P. Navas (2008), establecen la metodología para construir la Escala FUMAT que busca evaluar la calidad de vida en personas mayores y con discapacidad. Para su fundamentación teórica retoman las aportaciones de Schalock y Verdugo (2002/2003). Asimismo se llevó a cabo una exhaustiva revisión de la literatura científica que permitió la elaboración de un banco de 300 ítems con formato declarativo enunciados en tercera persona para ser contestados con una escala de frecuencia y que se organizaron de acuerdo según las dimensiones e indicadores de calidad de vida (Ver Tabla 1). Entre éstos, se seleccionaron 18-20 ítems para cada una de las ocho dimensiones $(\mathrm{N}=158)$.

Tomando a Muñoz Valdés, Poblete Toloza y Jiménez Figueroa (2012 p.209-210) la evaluación de la calidad de vida en familias con uno o más miembros con discapacidad es un tema central de los estudios interculturales (Verdugo, Córdoba \& Gómez, 2006), consensuando que su análisis no debería realizarse bajo una aproximación psicopatológica, sino más bien a través de una concepción en la que se prioricen las potencialidades y las capacidades de la familia (Tumbull, Blue-Banning, Turbiville \& Park, 1999; Tumbull, A.P. \& Tumbull, H.R., 2001). 
Tabla No. 1 Definición Operativa de la Calidad de Vida

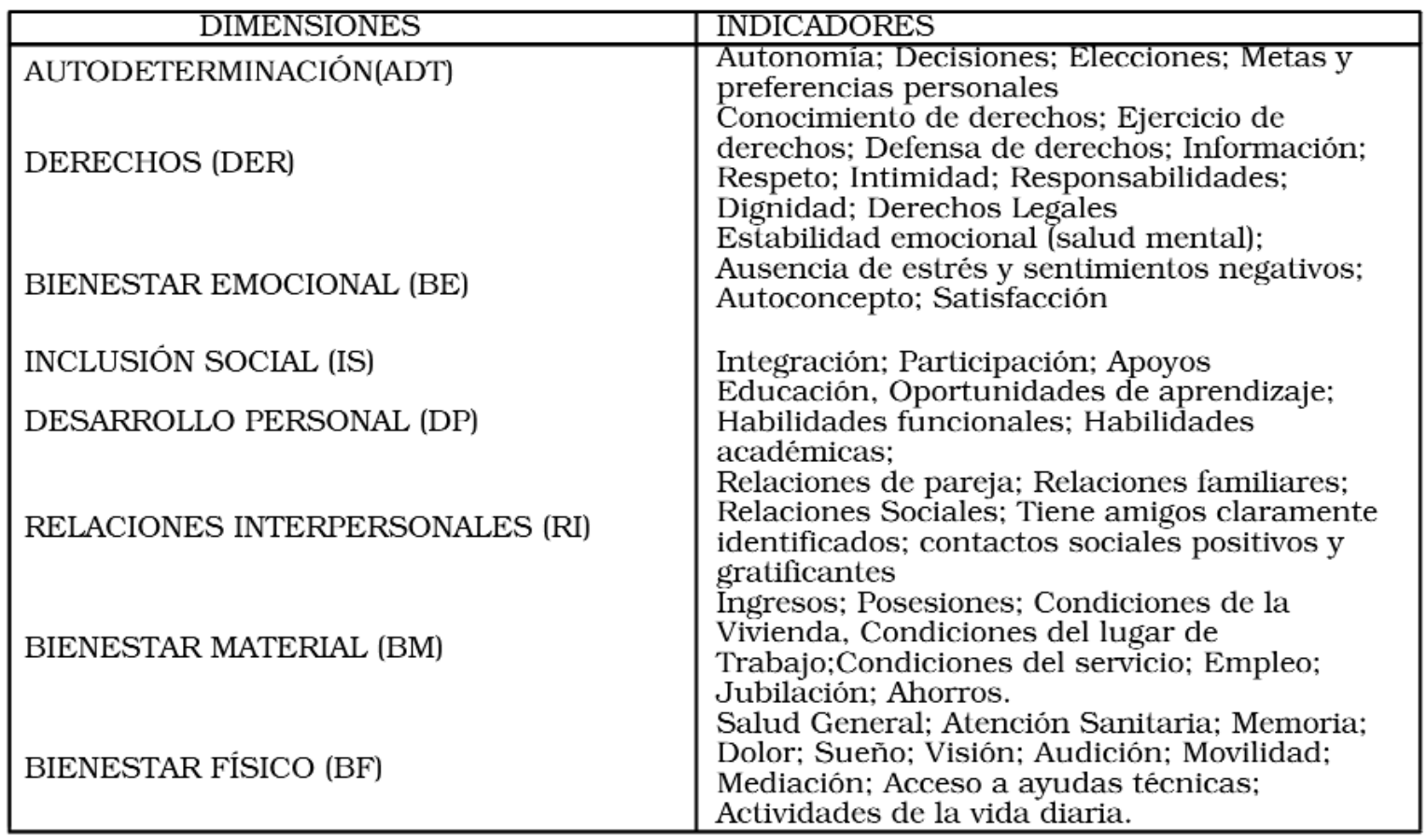

Cabe destacar que las limitaciones de un individuo tienen directa relación con el impacto de la deficiencia que presenta junto con las condiciones del entorno construido y las del entorno social. Así, el nivel de funcionamiento que una persona presenta en su vida depende de aquellos elementos de compensación y ajuste que están a disposición a partir de dicho contexto (Deaño, 2007). Además, es relevante destacar que el constructo actual de discapacidad está centrado en la expresión de limitaciones con respecto al funcionamiento individual dentro de un contexto social, lo que repercute en el sujeto como una desventaja que se inicia como una condición de salud con resultados de déficit en el cuerpo y sus estructuras (Schalock et al., 2007). (Yohanina Andrea Muñoz Valdés, Yohanna del Pilar Poblete Toloza y Andrés Eduardo Jiménez Figueroa, 2012 p.211)

$$
\text { Schalock y colaboradores }
$$

demostraron que la calidad de vida familiar se puede analizar independientemente de aquella centrada en la persona. Por ello, la calidad de vida es considerada como un concepto multidimensional (Zondek et al., 2006) (Yohanina Andrea Muñoz Valdés, Yohanna del Pilar Poblete Toloza y Andrés Eduardo Jiménez Figueroa, 2012)

Asimismo, tomando a (Ares, 2009) contar con el apoyo familiar es fundamental en el 
desarrollo de la socialización de las personas con discapacidad, puesto que es la familia el sistema directo en el cual se adquieren las primeras interacciones humanas (Muñoz Valdés, Poblete Toloza y Jiménez Figueroa, 2012, p.209-210) De igual forma, siguiendo a Mora et al., 2007) esto se aplica al mejoramiento de su calidad de vida, ya que cuanto mejores sean sus interacciones familiares, mayor será su nivel de calidad de vida y felicidad; mientras que ocurrirá lo contrario si las relaciones en sus hogares presentaran problemas y elementos más bien negativos, como un bajo apoyo y consenso familiar ante las exigencias que representa tener un integrante con discapacidad (Muñoz Valdés, Poblete Toloza y Jiménez Figueroa, 2012)

Siguiendo con estos autores, se observa que las familias de las personas con discapacidad se diferencian en sus características de aquellas que no tienen esta condición dentro de su núcleo, requiriendo atención psicosocial y orientación profesional para lograr una dinámica más armónica y positiva que favorezca el desarrollo de la persona con discapacidad (Valls, Vila \& Pallisera, 2004). Así, la calidad de vida de la familia se asocia al bienestar personal que va más allá de lo individual, alcanzando así a la comunidad (Mora, Córdoba \& Bedoya, 2007). (Yohanina Andrea Muñoz Valdés, Yohanna del Pilar Poblete Toloza y Andrés Eduardo Jiménez Figueroa, 2012)
Además, de acuerdo a Gine (2004) las personas con discapacidad tienen una participación social limitada, hay más elementos riesgosos que resultan como amenazas para su calidad de vida (Muñoz Valdés, Poblete Toloza y Jiménez Figueroa, 2012 p. 211)

Otro punto a considerar son otras variables que podrían ser consideradas en próximos estudios, tales como la inserción laboral y el apoyo social. Además, es importante considerar en futuras investigaciones el grado certero de discapacidad, los estándares de los niveles y las políticas públicas asociadas (Muñoz Valdés, Poblete Toloza y Jiménez Figueroa, 2012, p.214)

La calidad de vida es el resultado de la interacción entre la discapacidad de una persona y las variables ambientales que incluyen el medio físico, las situaciones sociales y los recursos $(\mathrm{MsC}$. Isabel Adela Vigil Zulueta,I Dr. C. Carlos Felipe Domínguez Eljaiek,I MsC. Marcia Sandra Hernández ZayasI y MsC. Carlos Ariel Domínguez OsorioII (2013 p.149).

3. Un acercamiento objetivo a la calidad de vida de las familias con integrantes con discapacidad

Como se ha apreciado en los párrafos anteriores, la calidad de vida es un concepto multidimensional que puede comprenderse tanto 
desde un punto de vista objetivo como subjetivo. En ese sentido, en este apartado solamente se tendrá una aproximación objetiva a la medición de la calidad de vida. Se ha descartado una aproximación subjetiva, ya que esta solamente se puede obtener de estudios de caso de corte micro y cualitativos, los cuáles prácticamente son inexistentes en México y no se diga en Jalisco.

En cuanto a la medición objetiva de la calidad de vida, se tomará uno de los criterios propuestos por Schalock y Verdugo (2002; 2008) denominado Bienestar Material (B.M.) el cual refiere los ingresos, posesiones materiales, condiciones de la vivienda, condiciones del lugar de trabajo, condiciones de los servicios que utiliza, empleo, jubilación y ahorro, como indicadores para medir la calidad de vida. En ese sentido - y por carencia de información - solamente nos enfocaremos a dos puntos. En el primero se expondrán los indicadores que existen sobre las personas con discapacidad en los Censos de Población y Vivienda y en el Consejo Estatal de Población Jalisco (COEPO) que son el empleo, la educación, la salud, vivienda y sus servicios. En el segundo - desde el punto de vista de sus derechos - se expondrá el diagnóstico que realizó en el 2012 la Comisión Estatal de Derechos Humanos de Jalisco, que establece en qué medida las instancias gubernamentales en Jalisco han cumplido los lineamientos de Convención sobre los Derechos de las Personas con Discapacidad, en particular, sobre los aspectos relacionados con la accesibilidad. A este respecto, estamos conscientes de que este es un acercamiento sumamente parcial a la calidad de vida de las personas con discapacidad en Jalisco: de ahí que se ha establecido que este trabajo es apenas una aproximación. Antes de exponer los dos puntos citados, se referirá cuantas personas con discapacidad hay en Jalisco y sus características.

3.1 Contexto general de la discapacidad en Jalisco

De acuerdo al censo del 2010, en Jalisco había 290,656 individuos que tienen una limitación física o mental en su persona y que les impide desarrollar sus actividades familiares, educativas y laborales de una forma óptima, y que por ende, se les considera como personas con discapacidad. Para el 2011 - de acuerdo al Consejo estatal de población de Jalisco-, esta cifra aumentó a 291 mil jaliscienses que tienen algún tipo de discapacidad, lo que representa el 4.0 por ciento de la población total de la entidad. De las personas con discapacidad el $50.3 \%$ son hombres y el $49.7 \%$ mujeres (Coepo, Jalisco 2011).

Asimismo, la posibilidad de padecer alguna limitación física o mental se incrementa con la edad. Por ejemplo, de los poco más de $52 \mathrm{mil}$ jaliscienses que tienen 85 años y más, el 49\% tiene alguna discapacidad; mientras que en el grupo de edad de 60 a 84 años hay 113 mil personas con discapacidad, lo que implica que el 18\% de las personas de este grupo de edad tienen alguna 
discapacidad. Aunque es de resaltar que hay $29 \mathrm{mil}$ niños de 0 a 14 años con alguna discapacidad y 31 mil jóvenes de 15 a 29 años (Coepo, Jalisco 2011).

En lo que respecta al tipo de discapacidad, se tiene que, del total de personas que conforman este grupo vulnerable, el 57.5\% tiene limitaciones para caminar o moverse; para ver aun utilizando lentes con un $23.3 \%$ y las personas que tienen algún tipo de limitación mental suman el 12.0\% del total. Por su parte la limitación para oír aun usando aparatos auditivos afecta al 10.3\%; hablar o comunicarse al 8.3\%; atender el cuidado personal al 5.4\% y poner atención o aprender al 5.3\%. Nótese que la suma de estos porcentajes no da el $100 \%$, debido a que el $14.5 \%$ de las personas con discapacidad los afecta más de una limitación (Coepo, Jalisco 2011).

En cuanto a las causas que originaron la discapacidad, el $38.0 \%$ se debe a enfermedades que posiblemente son de tipo crónico degenerativas y como segunda causa está la edad avanzada, con una frecuencia del $22.6 \%$. Las limitaciones desde el nacimiento representan el $17.8 \%$ de las discapacidades, y los accidentes generaron el 15.8\% (Coepo, Jalisco 2011).

3.2 El acceso al empleo, salud, educación, vivienda y servicios públicos

Empleo

Refiriéndonos al empleo de 268 mil personas de 12 años o más que tienen una discapacidad y que pueden trabajar, 71 mil trabajan, mientras que 4 mil están desocupados a pesar de estar buscando trabajo., es decir, el 26.5\% del total trabajan. De igual forma, la gran mayoría de estos empleos son de baja cualificación y con salarios precarios (Coepo, Jalisco 2011).

En cuanto a la población total de 7,090, 510 de 12 años o más que pueden trabajar, 3,055,636 trabajaban y 193, 384 estaban desocupadas (Consulta interactiva de datos, INEGI, 2010).

Si comparamos estas cifras, mientras que en la población total cerca del $43.1 \%$ de estas trabajan, dentro de las personas con discapacidad, el $26.5 \%$ lo hacen.

\section{Salud}

De 290 mil personas con discapacidad que había en Jalisco en el 2010, 194,035 tienen acceso a un servicio de salud predominantemente público (ya que de este total, sólo 3,488 van con médicos particulares) (Consulta interactiva de datos, INEGI, 2010) esto representa el $67.8 \%$ del total de las personas con discapacidad (Coepo, Jalisco, 2011)

De ellos, 39.3\% están afiliados al Seguro Social (IMSS), $22 \%$ al Seguro Popular o para una Nueva Generación, 3.2\% al ISSSTE, 1.8\% a una institución privada, $1.2 \%$ a otra institución no definida y $0.4 \%$ a Pemex, Defensa o Marina. Un dato interesante es que mientras el $3.1 \%$ de las personas sin discapacidad cubren sus necesidades

92 GOVELA, E.R.: Calidad De Vida En Las Familias Con Integrantes Con Discapacidad En Jalisco, Mexico: Una Aproximación Objetiva. 
médicas en instituciones privadas, las personas con discapacidad que también lo hacen, solamente son el $1.8 \%$.

Por su parte, los tipos de discapacidad que reportan menor porcentaje de derechohabientes son aquellos con dificultades mentales (57.9\%), para hablar o comunicarse $(61.7 \%)$ y poner atención o aprender (61.9\%), en cambio, las proporciones más altas se ubican en los individuos con dificultad para caminar o moverse (69.7\%), escuchar $(69.3 \%)$, ver $(68.8 \%)$ y atender el cuidado personal (64.5 por ciento) (Coepo, Jalisco, 2011).

En cuanto a los que son derechohabientes en la población total, encontramos que de 7,350,682 habitantes, 4,709,272 tienen acceso a un servicio de salud, lo que representa aproximadamente un 64\%.

\section{Educación}

En cuanto a su escolaridad, en el 2010, el $76.6 \%$ de las personas con discapacidad de 15 años en adelante saben leer y escribir, y $22.8 \%$ son analfabetas.

Para saber cuántas personas con discapacidad acceden a la educación especial en Jalisco y que infraestructura existe al respecto, se tomará como base el Censo de escuelas, maestros y alumnos de educación especial y especial 2013 que se encuentra en el Atlas Educativo de la página del INEGI. Para saber que tanta importancia se le ha dado al apoyo de la educación especial, respecto a la educación básica (a nivel preescolar y primaria) se remitirá a continuación el cuadro No. 1, 2 y 3 los cuáles exponen las escuelas, maestros y personal administrativo y alumnos existentes tanto en la educación básica como especial.

Cuadro No. 1: Escuelas De Educación Básica, Especial E Instancias De Apoyo Administrativo

\begin{tabular}{|c|c|c|c|c|}
\hline & $\begin{array}{l}\text { ESCUELAS DE } \\
\text { EDUCACIÓN } \\
\text { BÁSICA }\end{array}$ & APOYO ADMINISTRATIVO & $\begin{array}{ll}\text { ESCUELAS } & \text { DE } \\
\text { EDUCACIÓN } & \\
\text { ESPECIAL } \\
(\text { CAM })\end{array}$ & USAER \\
\hline PORCENTAJES & $\begin{array}{l}86.3 \% \text { de escuelas } \\
\text { de } \quad \text { educación } \\
\text { básica }\end{array}$ & 11.5.\% de instancias de apoyo administrativo & $1 \%$ de escuelas & $\begin{array}{l}1.2 . \% \text { de } \\
\text { ароуо }\end{array}$ \\
\hline CANTIDAD & $\begin{array}{l}\text { 15,348 de escuelas } \\
\text { e instancias de } \\
\text { apoyo } \\
\text { administrativo en } \\
\text { educación básica y } \\
\text { especial }\end{array}$ & & 153 & 183 \\
\hline
\end{tabular}


Fuente: Elaboración propia con base en el Censo de escuelas, maestros y alumnos de educación básica y especial, 2013.

En este primer cuadro, llama la atención que de los 15,348 centros educativos y de apoyo administrativo que existen en Jalisco para atender la educación básica y especial, solamente el 1\% de este total están dedicados a la educación especial, es decir, 153 Centros de Atención Múltiple (CAM) y existen 183 Centros de apoyo administrativo a la educación especial que son denominados USAER, lo que representa un $2.1 \%$ del total.

Cuadro No. 2: Maestros Y Personal Administrativo De Educación Básica Y Especial

\begin{tabular}{|c|c|c|c|c|}
\hline & $\begin{array}{l}\text { ESCUELAS DE EDUCACIÓN } \\
\text { BÁSICA }\end{array}$ & $\begin{array}{l}\text { APOYO } \\
\text { ADMINISTRATIVO }\end{array}$ & $\begin{array}{l}\text { ESCUELAS DE } \\
\text { EDUCACIÓN } \\
\text { ESPECIAL } \\
(\mathrm{CAM})\end{array}$ & USAER \\
\hline PORCENTAJES & $\begin{array}{l}87.9 \% \text { de maestros y } 9.2 \% \text { de } \\
\text { apoyo administrativo en } \\
\text { educación básica }\end{array}$ & $\begin{array}{l}9.2 \% \text { de personas } \\
\text { administrativo }\end{array}$ & $1.6 \%$ de maestros & $\begin{array}{l}1.3 . \% \text { de personal } \\
\text { administrativo }\end{array}$ \\
\hline CANTIDAD & $\begin{array}{l}\text { 130,460 de maestros y personal } \\
\text { administrativo de educación } \\
\text { básica y especial }\end{array}$ & & $\begin{array}{l}260 \text { maestros } y \\
\text { personal } \\
\text { administrativo }\end{array}$ & \\
\hline
\end{tabular}

Fuente: Elaboración propia con base en el Censo de escuelas, maestros y alumnos de educación básica y especial, 2013.

En este segundo cuadro se observa que de 130,460 maestros y personas administrativo que existen para apoyar la educación básica y especial, el $1.6 \%$ de este total, es decir, 260 dan clases y apoyan a la educación especial en Jalisco.

Cuadro No 3: Alumnos De Educación Básica Y Especial

\begin{tabular}{|l|l|l|}
\hline & $\begin{array}{l}\text { ALUMNOS DE } \\
\text { EDUCACIÓN } \\
\text { BÁSICA }\end{array}$ & $\begin{array}{l}\text { ALUMNOS } \\
\text { DE } \\
\text { EDUCACIÓN } \\
\text { ESPECIAL }\end{array}$ \\
\hline PORCENTAJES & $\begin{array}{l}56.2 \% \text { alumnos } \\
\text { de primaria y } \\
18.2 \% \text { preescolar }\end{array}$ & $0.5 \%$ alumnos \\
\hline CANTIDAD & $\begin{array}{l}1,677,821 \text { de alumnos } \\
\text { alumnos de } \\
\text { educación básica } \\
\text { yespecial }\end{array}$ \\
\hline
\end{tabular}

Fuente: Elaboración propia con base en el Censo de escuelas, maestros y alumnos de educación básica y especial, 2013. 
Finalmente en el cuadro vemos que de $1,677,821$ alumnos que fueron educados tanto en educación básica como especial, solamente el $0.5 \%$ de estos $(8,428)$ fueron atendidos en una escuela de educación especial en Jalisco.

De forma general, se puede apreciar, que los porcentajes en cuanto a escuelas, centros de apoyo administrativo, maestros y alumnos dedicados a la educación especial en Jalisco en cuanto a los niveles de preescolar y primaria, oscilan entre el $0.5 \%$ y el $1.6 \%$ por lo que el apoyo educativo a este sector poblacional es realmente mínimo.

Sin embargo, si contrastamos estos datos con lo que informa el Gobernador del estado de Jalisco Aristóteles Sandoval en su primer informe de actividades, en el 2012, en donde se afirma que se atendieron a 32,911 alumnos con discapacidad, encontramos que los 8,428 alumnos atendidos por los CAM representa un $32.9 \%$ de la cantidad informada por el gobernador.

Esta diferencia se debe probablemente a que en los CAM se atiende básicamente a niños y adolescentes que tienen múltiples tipos de discapacidades físicas e intelectuales, los cuáles por sus características- difícilmente se insertan en una escuela regular. A este respecto, hay muchos adolescentes y jóvenes con una discapacidad motora o sensorial (auditiva y visual) que al no tener ningún tipo de discapacidad intelectual no sólo pueden terminar su educación básica, sino media, media superior e incluso un posgrado. Sin embargo, esto nos habla de que no hay cifras exactas que nos digan exactamente cuántas personas con una limitación en su funcionamiento (de acuerdo a los criterios del censo del 2010) están estudiando, cuando de acuerdo al censo citado en el 2010, en Jalisco había 290,656. Si contrastáramos ese total con las 32,911 personas con discapacidad que accedieron a una escuela, veríamos que estos representan el 11.3\% del total de los que podrían estar estudiando.

En suma, tanto si consideramos a los niños y adolescentes con discapacidades graves que son atendidos en los CAM, que son 8,428 como los 32,911 que fueron a la escuela (en donde están incluidos los que asistieron a los CAM), aun así veríamos que el apoyo a la educación de las personas con discapacidad es marginal.

\section{Vivienda y servicios públicos}

En cuanto a la infraestructura urbana y el tipo de viviendas en donde viven las personas con discapacidad en Jalisco, observamos que de los 290,656, 287,048 viven en una vivienda particular, de las cuáles 273,242 viven en una casa independiente, 9,293 en un departamento, 2,401 en vecindades, 80 en cuarto de azotea, 49 en refugios y 117 en un local que no fue construido con fines habitacionales. En cuanto al número de cuartos que tienen las casas donde viven las personas con discapacidad, encontramos que 8,080 viven en construcciones que tienen un solo cuarto, 31,158 con dos cuartos y 70,875 con 3 cuartos. 
En cuanto al tipo de servicios con que cuentan esas viviendas, 3,533 personas con discapacidad habitan en viviendas que no disponen de luz eléctrica, y 8,428 no tienen drenaje. (Consulta Interactiva de Datos, INEGI, 2010).

En cuanto al tipo de vivienda, 1,801,306 de la población general vive en vivienda particular, de los cuáles 1,656,378 habita una casa independiente, 109,226 en departamento, 22, 685 en vecindad, 819 en cuarto de azotea. En cuanto al número de cuartos que tienen estas casas, situamos que 60,088 habitan una construcción que tiene un solo cuarto, 215,442 con dos cuartos y 467,940 con tres cuartos (Consulta interactiva de datos, 2010).

En cuanto al tipo de servicios con que cuentan esas vivienda 64,254 no tienen electricidad y 37,139 no tiene drenaje (Consulta interactiva de datos, 2010).

Mientras que $98.7 \%$ de las personas con discapacidad viven en una casa independiente (presumiblemente con familiares) y el 11.3\% restante viven en departamentos y vecindades, en la población total, el $91.9 \%$ viven en una casa independiente y el $8.1 \%$ vive en departamento, vecindades y cuartos de azotea. En cuanto a si tienen los servicios las habitaciones de las personas con discapacidad, aproximadamente el 1.2\% no tiene luz eléctrica y el 4.3.\% no tiene agua potable. En la población general, el 3.5\% no tiene luz y el $2.9 \%$ no tiene drenaje.
Refiriéndonos a cuantas personas viven por cuarto, tanto las PCD, como la población total, en un mayor porcentaje viven en viviendas con 3 cuartos.

\section{Conclusiones}

La calidad de vida de las personas con discapacidad de acuerdo a los autores consultados se puede medir considerando aspectos objetivos como subjetivos. En este trabajo se descartaron los objetivos por no contar con estudios cualitativos y en el contexto de Jalisco que nos permitieran determinar este concepto en base a estudios de caso.

En cuanto a la aproximación objetiva, se consideró únicamente el concepto de Bienestar Material de las PCD en Jalisco. A este respecto encontramos que en materia de empleo, solamente un $26.5 \%$ de estas personas que están en edad de trabajar lo hacen, frente a un $43.1 \%$ de las personas sin discapacidad. En cuanto al acceso al salud, el acceso a un servicio público, hay un porcentaje ligeramente más alto en favor de las PCD (un $67.8 \%$ frente a un $64 \%$ ).

En el ramo de la educación - y de acuerdo al Censo de escuelas, maestros y alumnos de educación especial 2013 - los porcentajes de instituciones, maestros, personas administrativos y alumnos que existen en los CAM y USAER que atienden básicamente a los alumnos con 
discapacidad intelectual (respecto a la educación básica) oscila entre un 0.6 a un 1.6\%) Sin embargo, si incluimos a los alumnos que tienen una discapacidad sensorial (sordos y sin habla) visual y motora que al no tener una discapacidad intelectual y por ende estudian desde la secundaria hasta la universidad, el porcentaje crece a un $11.3 \%$

En cuanto a la vivienda y los servicios públicos con que cuentan, se observa que cerca del 98.7\% de las PCD viven en una casa pero con un familiar que no tiene discapacidad y el resto $(11.3 \%)$ habitan en departamentos, vecindades o cuartos. En cuanto a las personas sin discapacidad, un $91.9 \%$ de estas viven en una casa propia y el $8.1 \%$ en departamentos, vecindades o cuarto.

En cuanto a la carencia de servicios de electricidad el 3.5\% de la población total no tienen esos servicios, respecto a un 1.2\% de las PCD. En cuanto al drenaje, el 2.9\% de la población total no tienen drenaje, respecto a un $4.3 \%$ y en cuantos cuartos viven, los porcentajes son muy similares: En cuanto al número de cuartos, ambos grupos, viven en un porcentaje mayor en viviendas de 3 cuartos al menos.

De forma general, en donde se visualiza una desventaja - para las PCD - es en el acceso al empleo $(26.5 \%)$ a la educación básica en su rama de educación especial (que en el caso de alumnos inscritos llega a un $0.5 \%$ ) pero que se incorpora a los alumnos de la discapacidad motora, sensorial y visual alcanza el 11.3\%. En cuanto a que vivan en una vivienda privada, estos alcanzan un $98.7 \%$ que se explica porque viven con sus familiares, frente a un $91.9 \%$ de las personas sin discapacidad. En cuanto a la deficiencia de los servicios de electricidad, drenaje y el número de cuartos en que viven, sus porcentajes son muy similares. En suma, los puntos en donde las PCD tienen mayores desventajas es en el acceso a la educación y al empleo.

Refiriéndonos a los programas existentes en el Estado de Jalisco que apoyan el desarrollo de las PCD, se observó que salvo el caso del programa del DIF llamado Desarrollo de Habilidades para la vida, que refiere el apoyo de la familia como un elemento primordial para la inclusión de las personas con discapacidad, todos los programas existentes refieren a las PCD como individuos aislados, como si partieran del supuesto que al apoyarlos a ellos de forma individual, por sí mismos, estos saldrán adelante.

En suma, si hablamos de igualdad de oportunidades entre las personas sin discapacidad y las personas con discapacidad, veríamos claramente que hay una notable diferencia entre ambos grupos, en donde las PCD ocupan un puesto marginal. En ese sentido, cuando se refirió el indicador objetivo del Bienestar material se comprobó que las posibilidades de acceder a una mejor calidad de vida por parte de las PCD es menor, sobre todo en cuanto al acceso a la educación y el empleo. De igual forma, sigue prevaleciendo la visión de la discapacidad como 
enfermedad que se "cura" con una rehabilitación; asistencialista en cuanto a que dándole unos cuantos apoyos el gobierno ha cumplido su compromiso, cuando esto solo es una estrategia que resuelve el problema de momento, pero no se atacan las causas que provocan que las personas con discapacidad no se pueden integran cabalmente a la sociedad e individualista.

Justamente este último punto nos habla de que no se considera el hecho primordial de que las personas con discapacidad que han logrado tener acceso a una educación y aun posterior trabajo, ha sido gracias al fuerte apoyo que ha recibido de la familia. En ese sentido, si las familias recibieron un apoyo más fuerte del Gobierno del Estado y de empresas privadas y de la sociedad civil organizada, habría más personas con discapacidad productivas.

\section{Referencias}

VERDUGO A., Miguel Ángel, Como mejorar la calidad de vida de las personas con discapacidad: Instrumentos y estrategias de evaluación. Salamanca, Amaru , 2006.

\section{Referencias electrónicas}

ANTUNEZ F, María Eugenia y Balcazar de la Cruz, Andrés (2005) Diagnóstico sobre la discapacidad en México. In: Organización de Estados Americanos, 2005. http://scm.oas.org/pdfs/2007/DIL00140s.pdf

ARDILLA, Ruben Calidad de vida: Una definición integradora. Revista Latinoamericana de psicología $\quad$ Vol. 2003. http://www.redalyc.org/pdf/805/80535203.pdf

Encuesta Nacional sobre Discriminación en México (ENADIS, 2010). México: CONADIS. http://www.conapred.org.mx/userfiles/files/En adis-PCD-Accss.pdf

COEPO. En Jalisco hay 291 mil personas con algún tipo de discapacidad. domingo 5 de junio del 2011.

http://coepojalisco.blogspot.mx/2011/06/enjalisco-hay-291-mil-personas-con.html CORDOVA A., Leonor; Gómez Benito, Juana, y Verdugo Alonso, Miguel Ángel, Calidad de vida familiar en personas con discapacidad: Un análisis comparativo. Universitas psychologicas V.7 No. 2 Mayo - Agosto 2008, Pontificia Universidad Javeriana, Bogota, Colombia. http://www.redalyc.org/pdf/647/64770206.p $\underline{\text { df }}$

GOMEZ V., Maria, y N. Sabeh, Eliana. Calidad de vida.

evolución del concepto y su influencia en la investigación y la práctica. Instituto Universitario de Integración en la Comunidad, Facultad de Psicología, Universidad de Salamanca.

98 GOVELA, E.R.: Calidad De Vida En Las Familias Con Integrantes Con Discapacidad En Jalisco, Mexico: Una Aproximación Objetiva. 
http://campus.usal.es/ inico/investigacion/inve sinico/calidad.htm

INEGI Atlas Educativo. Censo de escuelas, maestros y alumnos de educación básica y especial, México, 2013. http://cemabe.inegi.org.mx/

INEGI. Consulta interactiva de datos., México http://www.inegi.org.mx/est/lista cubos/

INEGI. Censo de Población y Vivienda 2010. Base de datos de la muestra censal, México: INEGI., $\quad$ México, 2010. http://www.inegi.org.mx/

MUÑOZ V., Yohanina Andrea; Poblete Toloza, Yohanna del Pilar y Jiménez Figueroa, Andrés. Calidad de vida familiar y bienestar subjetivo en jóvenes con discapacidad intelectual de un establecimiento con educación especial y laboral de la Ciudad de Talca in Interdisciplinaria vol.29 no.2 Ciudad Autónoma de Buenos Aires ago./dic. 2012, http://www.scielo.org.ar/scielo.php?script $=$ sci a rttext\&pid $=$ S1668-70272012000200001

Revista Iberoamericana de educación. Monográfico No. 63, Calidad de vida en el ámbito de la educación de personas con discapacidad, septiembre - diciembre 2013.

SANDOVAL, Aristoteles. Primer Informe de Gobierno. Informe de programas operativos anuales. Tomo II, 2014
VIGIL Z, Isabel Adela, Domínguez Eljaiek C. Carlos Felipe, Hernández Zayas, Marcia Sandra y Domínguez Osorio, Carlos Ariel. Enfoque bioético de la discapacidad y calidad de vida, in: biblioteca virtual de cuba. http://bvs.sld.cu/revistas/san/vol17 1 13/san1 $\underline{81713 . h t m}$ 